Auslaufmodell macht. Zum anderen, indem sie selbst bei der am 18. April 2013 hitzig geführten und von Zwischenrufen begleiteten Plenardebatte das Scheitern der 2001 beschlossenen Vereinbarung zur Förderung der Chancengleichbeit in der Privatwirtschaft zwischen der Bundesregierung und den Spitzenverbänden der deutschen Wirtschaft als Argument für das frühere Versagen der Opposition heranzieht. Die Erfahrung, dass reine Lippenbekenntnisse der Unternehmen keine Veränderung in Vorständen und Aufsichtsräten anstößt, wurde somit schon einmal und mehr als deutlich gemacht. Und obwohl die Union das Problem des fortwährenden Stillhalteabkommens zulasten der Frauen zumindest als probates Mittel zur Anklage der Opposition erkannt hat, setzt sie leider noch weitere sieben Jahre auf die Selbstregulierungskräfte des Marktes. Dennoch bleibt offen, ob über die bloße Absichtserklärung hinaus die Quote kommt. Mit dem Wunschkoalitionspartner FDP ist sie bisher nicht zu machen. Selbst die Flexi-Quote sollte ursprünglich in diesem Jahr gesetzlich festgeschrieben werden. Dieser Teil des Stufenplans wurde dann kurzerhand und mit bis heute ausbleibender Begründung auf die Zeit nach der anstehenden Wahl vertagt. Und Wahlprogramme sind weder Garant für eine Wiederwahl noch Garant für Gesetze. Das Thema Chancengleichheit muss zwingend auf der politischen Agenda bleiben. ${ }^{8}$ Den öffentlichen Druck hierfür können, wie die Märchenstunde im Parlament am 18. April 2013 gezeigt hat, nur die Aktivistinnen und Aktivisten in der Zivilgesellschaft herstellen.

8 S. auch: Wahlprüfsteine zur Bundestagswahl 2013 für das Arbeits-, Wirtschafts- und Gleichstellungsrecht (K1) in dieser Ausgabe, S. 55.

\title{
djb-Initiative bringt mehr „Frauen in die Roten Roben“
}

\section{Eva Schübel}

Vizepräsidentin des djb; Bundesanwältin beim BGH, Karlsruhe

Bei der Richterwahl am 21. März 2013 - der zweiten Runde nach Beginn unserer Initiative im Herbst 2011 - hat es wieder ein Auf und Ab der Emotionen gegeben. Jubel wegen des hervorragenden Ergebnisses für den Bundesgerichtshof (sechs von acht Stellen an Kandidatinnen) und Freude wegen einer Zweidrittelmehrheit beim Bundesarbeitsgericht - dagegen lange Gesichter im Übrigen: für den Bundesfinanzhof wurden zwei Richterinnen und fünf Richter und für das Bundessozialgericht eine Richterin und 4 Richter gewählt; das Bundesverwaltungsgericht ist wieder einmal Schlusslicht mit einem Männerduo. Aufs Ganze gerechnet liegt die Frauenquote bei 44 Prozent. Das sehr gute Ergebnis für den Bundesgerichtshof relativiert sich, wenn man berücksichtigt, dass im vergangenen Jahr zehn Richter und nur zwei Richterinnen gewählt worden sind. Rechnen wir 2012 und 2013 zusammen, ergibt dies für den Bundesgerichtshof 12 neue Richter und acht neue Richterinnen.

Wir müssen daher zu erreichen suchen, dass bei jeder künftigen Wahl für jedes Bundesgericht mindestens 50 Prozent Frauen gewählt werden. Anderenfalls wird der Anteil der Bundesrichterinnen erst in mehr als 20 Jahren hälftig sein, was inakzeptabel ist.

Besser sieht es bei den Wahlvorschlägen aus. Unsere Präsidentin hat jedes Treffen mit Präsidentinnen und Präsidenten der Oberlandesgerichte und der Bundesgerichte sowie den Leitungen der Justizministerien genutzt, das Gleichstellungsdefizit bei den obersten Bundesgerichten aufzuzeigen und unser Anliegen zu verdeutlichen. Zur Erinnerung: Bei der Wahl 2011 war die Zahl der weiblichen Kandidaten auf einem deprimierenden Tiefstand angelangt. Unter 23 Kandidaten für den Bundesgerichtshof befanden sich gerade drei Frauen sowie jeweils nur eine Frau unter 17 Bewerbern für das Bundesverwaltungsgericht und unter sieben Bewerbern für den Bundesfinanzhof. Dieser Befund veranlasste mich zu der
Initiative „Frauen in die Roten Roben“, die bereits 2012 Wirkung gezeigt hat. Es werden zunehmend mehr Frauen vorgeschlagen. Im letzten Jahr waren unter den Kandidaten für den Bundesgerichtshof ein Drittel Frauen. In diesem Jahr waren es sogar 19 Richterinnen von 37 Kandidaten (=51,5\%!). Das Schlusslicht nicht nur bei der Wahl selbst bildete das Bundesverwaltungsgericht mit einer Vorschlagsquote von nur 20 Prozent. Sehr erfreulich ist, dass überproportional viele weibliche Neuvorschläge Parteien zuzurechnen sind, die das Thema Gleichstellung erst seit Kurzem ernster nehmen. Gleiche Wahlchancen für Richterinnen bestehen freilich erst dann, wenn auf den Wahllisten für die einzelnen Bundesgerichte je zur Hälfte Frauen und Männer stehen.

Hauptsächlich bestücken die Länderjustizministerien bzw. diejenigen Ressorts, zu deren Geschäftsbereich der jeweilige Gerichtszweig gehört, die Wahllisten. Denn die im Richterwahlausschuss vertretenden Abgeordneten machen von ihrem Vorschlagsrecht eher zurückhaltend Gebrauch. Die Auswahl der Kandidatinnen und Kandidaten ist in den Ländern offensichtlich Chefsache. Jedenfalls werden die Stellen, die ein Bundesland bei den Bundesgerichten besetzen kann, selten bekannt gemacht oder gar ausgeschrieben. Die zuständigen Ministerien führen interne Listen und entscheiden in der Regel ohne die Beteiligung der Gleichstellungs- bzw. Frauenbeauftragten und Richtervertretungen, wer gelistet und schließlich für ein Bundesgericht vorgeschlagen wird. Ein durch und durch intransparentes Verfahren, das vor allem Interessenten mit guten Kontakten in die Hausleitungen der Ministerien und Oberlandesgerichte nützt. Hier gilt es anzusetzen.

Über das Netzwerk der Gleichstellungs- bzw. Frauenbeauftragten und -vertreterinnen der Landesjustizverwaltungen habe ich den Kontakt in die Länderjustiz gesucht. Dabei hat mir der Umstand, dass ich selbst die Gleichstellungsbeauftragte des Generalbundesanwalts bin, die Türen zu den Kolleginnen geöffnet. Dies erleichtert auch meine Teilnahme an NetzwerkTreffen, bei denen ich den Weg in die obersten Bundesgerich- 
te aufzeige. Ich diskutiere mit den Kolleginnen die vielfältigen Einflussmöglichkeiten der Gleichstellungsbeauftragten. Es beginnt schon damit, dass der Sprung an ein oberstes Bundesgericht neben der hohen fachlichen Qualifikation in der Regel eine R 2-Position voraussetzt. Ein solches Beförderungsamt ist jedoch gerade für Richterinnen und Staatsanwältinnen, die selbst Kinder betreuen, schwierig zu erreichen. Kinder sind der Karrierekiller Nr. 1, dies ist das Ergebnis der NRW-Studie „Frauen in Führungspositionen der Justiz“ aus dem Jahr 2011.

Die Gleichstellungsbeauftragten müssen daher bei den Beförderungschancen ansetzen. Sie müssen versuchen, ein transparentes, Chancengleichheit wahrendes Verfahren und eine geschlechtergerechte Vergabe der Sprungbrettpositionen zu erreichen. Mit Hilfe ihres Initiativrechts können sie zusätzliche Karrierewege für Richterinnen und Staatsanwältinnen mit Familienpflichten aufzeigen. Sie können Auskunft über die Beurteilungsnoten verlangen und prüfen, ob der Vergleich von beurteilten Frauen und Männern sowie Vollzeit- und Teilzeitbeschäftigten Verzerrungen aufdeckt. Sie dürfen Richterinnen und Staatsanwältinnen über die Karrieremöglichkeiten informieren und sie bei Bewerbungen unterstützen. Eine erfolgreiche Abordnung an ein oberstes Bundesgericht als wissenschaftliche Mitarbeiterin erhöht die Chance, als Bundesrichterin dorthin zurückzukehren. Schließlich sollten die Gleichstellungsbeauftragten einfordern, an den Entscheidungen beteiligt zu werden, wer auf die internen Vorschlagslisten aufgenommen und wer letztendlich vorgeschlagen wird. Die Gleichstellungsbeauftragten gewinnen zudem umso mehr an Einfluss, je mehr sie sich mit ihren Kolleginnen in den verschiedenen Instanzen vernetzen und regelmäßig fachlich austauschen.

Auf Bundesebene hat sich ein Arbeitskreis der Gleichstellungsbeauftragten der Bundesgerichte etabliert, der auch die
Kolleginnen des Bundesverfassungsgerichts, der Bundesanwaltschaft, des Bundesministeriums der Justiz und des Bundesministeriums für Arbeit und Soziales, das für die Wahlen zum BAG und BSG zuständig ist, einschließt. Ein wichtiges Thema ist, die Beteiligung der Gleichstellungsbeauftragten an den Sitzungen des Präsidialrats zu erreichen, bei dem sich die Kandidatinnen und Kandidaten für die Richterwahlen vorstellen müssen.

Neben der Neuen Richtervereinigung (NRV), die unsere Initiative „Frauen in die Roten Roben“ von Anfang an unterstützt, hat sich inzwischen der Bund der Richterinnen und Richter, Staatsanwältinnen und Staatsanwälte (DRB) des Themas angenommen und eine Arbeitsgruppe gebildet. Die fehlende Transparenz der Bundesrichterwahlen benachteiligt ja auch potentielle männliche Kandidaten. Unser Ansatzpunkt ist jedoch ein anderer: Wir brauchen auf dem gesamten Weg in Führungspositionen der Justiz Chancengleichheit für Frauen. Das über Jahrzehnte gepflegte Argument, es gebe nicht genügend qualifizierte Frauen, ist ausgeräumt. Betrachten wir nur die nachwachsende Frauenpower auf der einen und die geringe Zahl an jährlich zu besetzenden Führungsstellen auf der anderen Seite. Gab es 2011 nur drei Frauen auf der Wahlliste für den Bundesgerichtshof, hat man in diesem Jahr 19 Kandidatinnen präsentieren können. Die Auffassung „Wer suchet, der findet“ vertritt der djb auch im ähnlich gelagerten Projekt „Frauen in die Aufsichtsräte“. Unsere beiden Initiativen beginnen den Blickwinkel in Politik und Gesellschaft dahin zu verändern, dass Gleichstellung eben nicht von selbst kommt. Artikel 3 Absatz 2 des Grundgesetzes weist die staatlichen Repräsentanten seit annähernd 20 Jahren daraufhin, dass sie die Gleichberechtigung von Frauen und Männern tatsächlich durchsetzen müssen.

\section{Werden Beamtinnen bei der Beurteilung benachteiligt?}

\section{Dr. Karin Tondorf}

Mitglied der Kommission Arbeits-, Gleichstellungs- und Wirtschaftsrecht des djb; Freie Wissenschaftlerin/Beraterin, Seddiner See

Will eine Beamtin im öffentlichen Dienst aufsteigen, muss sie zu den Besten gehören. Ihre Chancen stehen gut, wenn sie eine Beurteilung vorweisen kann, die ihr eine sehr gute, wenn nicht gar eine hervorragende Leistung und eine entsprechende Eignung und Befähigung für die zukünftige Stelle bescheinigt. Doch wie verhält es sich mit der Chancengleichheit von männlichen und weiblichen Beamten, wenn es um die Beurteilung und Beförderung geht? Inwieweit können Beamtinnen (und Beamte) darauf vertrauen, dass ihre Leistungen angemessen und diskriminierungsfrei beurteilt werden?

Diese Frage stellten sich die Wissenschaftlerinnen Andrea Jochmann-Döll und Karin Tondorf ${ }^{1}$ bezogen auf den Polizeivollzugsdienst in Deutschland. Dieser Bereich ist bis heute eine Beschäftigungsdomäne von Männern geblieben, obwohl immer mehr Frauen bei der Kriminal-, Schutz- und Bereitschaftspolizei ihren Dienst tun. Seit geraumer Zeit hegen Frauen in der Gewerkschaft der Polizei (GdP) sowie Gleichstellungsbeauftragte den Verdacht, dass Frauen und Teilzeitbeschäftigte bei der Beurteilung und Beförderung benachteiligt sein könnten. Es gab jedoch nur wenige aussagekräftige Beurteilungsstatistiken, auf die sie sich berufen konnten.

Mit der neuen wissenschaftlichen Untersuchung liegen nun umfangreiche Daten über Beurteilungsergebnisse von Frauen und Männern im Polizeivollzugsdienst vor: 12 Bundesländer und die Bundespolizei übersandten den Wissenschaftlerinnen ihre statistischen Beurteilungsdaten sowie die jeweils geltenden Beurteilungsrichtlinien zur Analyse. Darüber hinaus stellten sich betriebliche Expert/inn/en für Interviews zur Verfügung.

1 Jochmann-Döll, Andrea/Tondorf, Karin (2013): Nach Leistung, Eignung und Befähigung? Beurteilung von Frauen und Männern im Polizeivollzugsdienst, gefördert von der Hans-Böckler-Stiftung, Arbeitsheft 276, Düsseldorf. 